\title{
Transport in ratchets with single-file constraint
}

\author{
C. M. ARIZMENDI ${ }^{1}$ and F. FAMILY $Y^{2, *}$ \\ ${ }^{1}$ Instituto de Investigaciones Científicas y Tecnológicas en Electrónica (ICYTE), Facultad de Ingeniería, \\ Universidad Nacional de Mar del Plata, Av. J.B. Justo 4302, 7600 Mar del Plata, Argentina \\ ${ }^{2}$ Department of Physics, Emory University, Atlanta, GA 30322, USA \\ *Corresponding author. E-mail: Phyff@emory.edu
}

\begin{abstract}
Diffusion of particles in restricted geometries is a subject of considerable interest due to their applicability in the observation and manipulation of nanoscopic systems. The single-file model has been introduced to model situations where the particles are constrained to move in nanopores in a single file without the chance for a particle exchange. Similarly, the motion of Brownian particles moving in a ratchet potential has been an active area of research because of its applicability to a wide range of nanoscale systems, including the transport properties of molecular motors. Here, we briefly review these models and discuss particle motion in a ratchet model constrained with the single-file condition. The motion of overdamped particles in a single-file model constrained to move in a ratchet potential is subdiffusive with mean square displacement that has $t^{\frac{1}{2}}$ time dependence when hard-core interaction is significant, and becomes diffusive for long times. The $N$-particle switching time for maximum current is $N$ times the switching time for one particle maximum current.
\end{abstract}

Keywords. Single file interaction; thermal ratchet; anomalous diffusion.

\section{PACS Nos 87.15.Aa; 87.15.Vv; 05.60.Cd; 05.45.Ac}

\section{Introduction}

Models suitable to account for the single-particle diffusional mechanisms in constrained-flow geometries have been the subject of increasing attention due to the exponential increase in the direct observation and manipulation of nanoscopic systems. This type of process [1] has been reported in transport of adsorbate molecules through nanoporous materials [2], in collective motion of ions through biological channels and membranes [3], in sliding of proteins along DNA [4] molecules as well as in nanodevices and cellular flows [5].

Hard-core interaction appears when the pore or channel is so narrow that particles cannot pass each other. In other words, the longitudinal motion of each particle is hindered by the presence of its neighbors, which act as moving obstacles. This hard-core diffusion process is also known as "single-file diffusion" in the literature.

Anomaly in protein molecule diffusion on a cell membrane has been associated with the crowding produced by the presence of fixed and mobile obstacles in a stochastic random walk model [6]. Diffusion was found to be highly anomalous when the obstacles are fixed, though randomly distributed. On the other hand, collision with picket fences [7], membrane proteins anchored to the membrane skeleton that function as diffusion barriers in the cell membrane, did not contribute to anomalous diffusion. The effects of lipid microdomains (also known as lipid rafts) covering a significant area of the membrane with excluded proteins have a large effect on the anomalous exponent.

In one dimension, the single-file (SF) model consists of $N$ unit-mass particles constrained to move along a line following a specified dynamics. The hard-core interaction forbids mutual exchanges between the particles. This is the single-file condition; i.e., the order of the diffusing particles is conserved. This kind of interaction alters the Brownian motion [8-10]. The particle motion is subdiffusive and the mean-square displacement grows with time as $t^{\frac{1}{2}}$, while the center of mass of the system of particles exhibits normal diffusion. Subdiffusion is related to anticorrelation of the jump sequences in a single-file model [11]. Three temporal regimes and the corresponding time scales - ballistic, diffusive, and subdiffusive - are obtained in a Langevin simulation for single-file diffusion [12].

On the other hand, fluctuation-driven transport in the biased Brownian particle motion, widely known as the ratchet model [13-15] can also exhibit anomalous diffusive transport and is used to model the motion 
of particles and macromolecules in a wide range of phenomena, including transport in biological intra-cell molecular motors, particle separation and the design of nanoscale molecular motors and pumps. Ratchets with quenched disorder tracks exhibit several scaling regimes with anomalous transport properties [16]. Long-range correlated spatial disorder has also been found to produce anomalous diffusion in an overdamped rocking ratchet [17]. A trapping mechanism is proposed as the origin of the anomalous behavior in transport properties of overdamped ratchets with disorder [18].

The collective behavior of particles that move on a finite line shows [19] a net current that depends on the density of particles. A very interesting stochastic resonance effect on rectification is obtained at a given temperature in a study of fluctuating tilt Brownian ratchets based on fractional subdiffusion in sticky viscoelastic media [20]. The rectification effect vanishes in the adiabatically slow modulation limit and optimizes in a driving frequency range. In other related work on flashing ratchets [21] a finite subdiffusive current is found at zero temperature for random flashing and for periodic flashing for a certain frequency window.

It would be interesting to investigate the effect of the single-file interaction on transport properties of the Brownian ratchet. In this paper we consider this problem using a numerical simulation of ratchets in which the associated Langevin equation is converted into a jump process [14]. We have already used this algorithm to study the approach to the steady state in Brownian ratchets [22], the memory correlation effect on ratchets [23], and to calculate the algorithmic complexity in the ratchet motion [24].

\section{The model}

The model for ratchets that we have used is based on the model proposed by Astumian and Bier [15, 22, 23]. We use the on-off version of Astumian and Bier model that consists of an asymmetric piecewise linear potential where the barrier potential is switched between the on and off states. The asymmetry parameter $\alpha=10 / 11$ and the maximum potential $V_{0}=1$ are the same as in Refs $[22,23]$. The fluctuations of the barrier height can produce a net flow even with the net macroscopic force that is zero at all times. The general Langevin equation describing the ratchets that we will consider is

$\dot{x}=F_{ \pm}(x)+\sqrt{2 k_{B} T} \xi(t)$,

where $T$ is the temperature, $k_{B}$ is the Boltzmann constant, and $\xi(t)$ represents the Gaussian noise term with delta function correlation $\left\langle\xi(t) \xi\left(t^{\prime}\right)\right\rangle=\delta\left(t-t^{\prime}\right)$. The on-off force $F_{ \pm}(x)$ is applied to the particle through the asymmetric piecewise linear on-off potential.

In our numerical simulations we have followed the approach of Elston and Doering [14] with the introduction of the single-file interaction constraint. In this approach, the variable $x$ that represents the position of the ratchet walker is discretized, converting the Langevin equation into a jump process. The master equation for the jump process, transition rates and other simulation characteristics may be found in Refs [14, 22, 23].

The additional requirement for the single-file condition is

$x_{1}<x_{2}$,

$x_{i-1}<x_{i}<x_{i+1}, 1<i<N$,

$x_{N-1}<x_{N}$,

where $N$ is the number of particles considered.

\section{The dynamics of the SF particles}

As in previous papers $[25,26]$ the transport properties are determined using cumulants. The single-file condition on a free particle moving in a noisy environment modifies the usual Brownian motion [8-10] making the particle behave in a subdiffusive way: the mean square displacement grows with time as $t^{\frac{1}{2}}$. We calculated the net current, associated with the first order cumulant and the second order cumulant in the flashing ratchet model with single-file interaction between particles in order to see if the features of the diffusion process are altered by the ratchet motion.

We have studied the evolution of an ensemble of $N$ single-file interacting particles, uniformly distributed over one whole potential valley. The initial distance between particles does not affect the net current or the mean square displacement evolution. The only difference is that the time spent in reaching the single-file characteristic mean square displacement time dependence depends on the initial interparticle distance, as expected.

The simulations were carried out over 1000 trajectories of typically $10^{5}$ to $10^{6}$ time steps for every one of the $N$ particles. We set $k_{B} T=1$ and use a stepsize of $\Delta x=0.01$.

The diffusion behavior may be seen in figure 1 for $N=50$ and $f=4$ where the evolution of the cumulant $C_{2}(t)=\left\langle(x(t)-\langle x(t)\rangle)^{2}\right\rangle$ as a function of time is shown. For short times the diffusion is highly impeded by the neighbour particles and the diffusion scales with an exponent smaller than $1 / 4$. The hard-core interaction effect on the particle spreading appears at intermediate times with the characteristic scaling $t^{\frac{1}{4}}$. At 


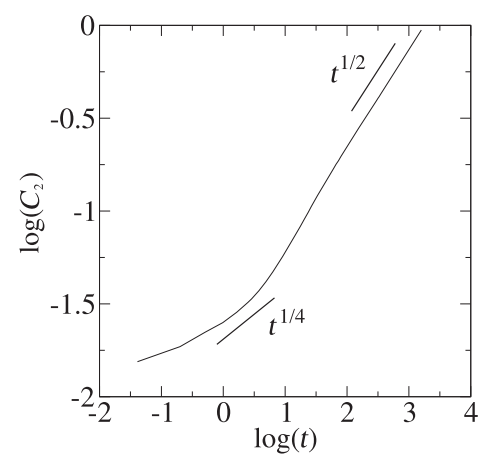

Figure 1. Second order cumulant as a function of time for on-off frequency $f=4$ and number of particles $N=50$. Three different scaling behaviors may be noticed: For short times the neighboring particles constrain the diffusion with a scaling exponent less than 1/4. At intermediate times the hardcore interaction effect on the particle spreading appears, with the characteristic scaling $t^{\frac{1}{4}}$. At longer times the particles are far enough from each other to interact and the motion tends to normal diffusion.

long times, particle motion reverts to normal diffusion because neighboring particles are far from each other and do not interact. The different scaling behaviors are shown in figure 1.

The variation of the current with the switching frequency is shown in figure 2 , for $N=50$. It has a similar form as the relation between the current and the frequency in single particle flashing ratchet [15] except that the frequency at which the maximum current occurs is different.

The number of SF particles $N$ as a function of the on-off frequency $f$ where maximum net current and normal diffusion appear are shown in figure 3. The frequency where maximum current occurs decreases with increasing number of particles, while one particle ratchet maximum current frequency [15] appears as the frequency limit for low number of particles.

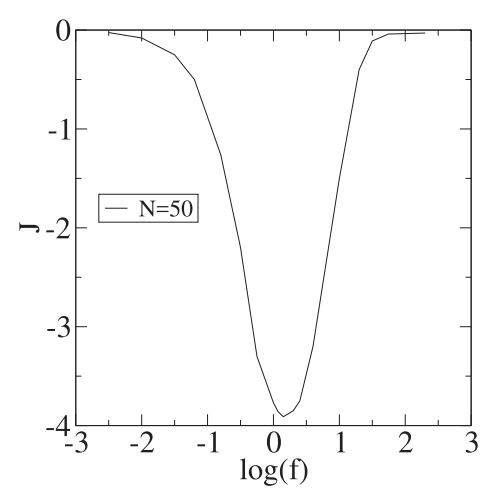

Figure 2. Net current $J$ for $N=50$ particles as a function of the on-off frequency $f$.

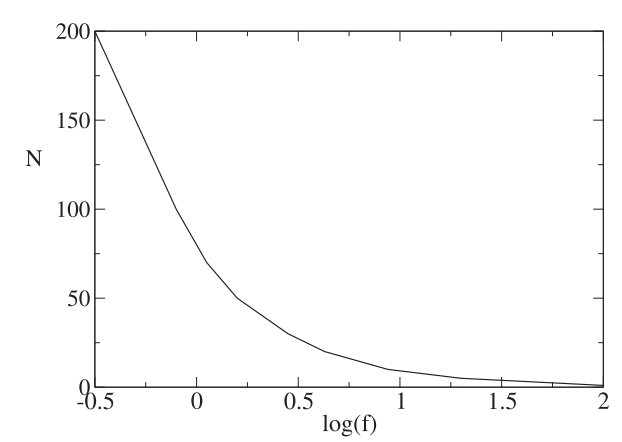

Figure 3. Number of SF particles $N$ as a function of the onoff frequency $f$ with maximum net current. The curve tends to one particle ratchet maximum current frequency for small number of particles.

Astumian and Bier [15] attribute the maximum current with the time for adiabatic adjustment on the steeper slope $\frac{(1-\alpha)^{2}}{2}$. When $N$ hard-core interacting particles are moving on the flashing ratchet the corresponding time for adiabatic adjustment increases because the motion of a particle depends on the other particles. An estimate of the time for adiabatic adjustment on the steeper slope for $N$ particles is $N$ times the time for one particle. This estimate corresponds well with the result shown in figure 3.

\section{Conclusions}

In this paper we discussed the problem of particle motion in constrained flow geometries. This is a subject of increasing interest due to the rapid growth in direct observation and manipulation of nanoscopic systems. When hard-core interactions are present, such as in flows in pores or channels, particles cannot pass each other and the motion of each particle is hindered by the presence of its neighbors. This is known as "single-file diffusion". In one dimension, the single-file model consisting of particles that are constrained to move along a line have been studied [8-10]. It is well established [8-10] that in the presence of single-file condition, particle motion is subdiffusive. On the other hand, fluctuation-driven transport in the ratchet model [13-15] can also exhibit anomalous diffusive transport. Here, we investigated the effect of the single-file interaction on transport properties of the Brownian ratchet. We calculated the drift current and the diffusion of a variable number $N$ of overdamped singlefile interacting particles in an on-off ratchet potential in a one-dimensional infinite system. We found that the characteristic scaling $t^{\frac{1}{4}}$ for diffusive single-file interacting particles appears at intermediate times. At longer times, in the infinite one-dimensional system, the particles drift far from each other and the interaction between 
neighboring particles fades away and the motion reverts to normal diffusion. The net current depends on the onoff switching frequency in the same way as in the one particle on-off ratchet model. The difference is that for a system of $N$ particles, the characteristic time for maximum current is $N$ times the characteristic time for a single particle. This is related to the fact that asymptotically the system is composed of $\mathrm{N}$ non-interacting particles.

\section{Acknowledgements}

This work was partially supported by Universidad Nacional de Mar del Plata.

\section{References}

[1] H Scher, M F Shlesinger and J T Bendler, Phys. Today 44, No. 1, 26 (1991); M F Shlesinger, G M Zaslavsky and J Klafter, Nature (London) 363, 31 (1993); J P Bouchaud and A Georges. Phys. Rep. 195, 127 (1990)

[2] J Kärger and M Ruthven, Diffusion in Zeolites and in Other Microporous Solids (Wiley, New York, 1992)

[3] D J Aidley and P R Standfield, Ion Channels: Molecules in Action (Cambridge University Press, New York, 1996)

[4] A Bakk and R Metzler, J. Theor. Biol. 231, 525 (2004)

[5] B Alberts et al., Molecular Biology of the Cell, 6th edition (Garland, New York, 2014)

[6] D V Nicolau, J F Hancock and K Burrage, Biophys. J. 92, 1975 (2007)

[7] C Nakada, K Ritchie, Y Oba, M Nakamura, Y Hotta, R Iino, R S Kasai, K Yamaguchi, T Fujiwara and A Kusumi, Nat. Cell Biol. 5, 626 (2003)
[8] T E Harris, J. Appl. Probab. 2, 323 (1965)

[9] J L Lebowitz and J K Percus, Phys. Rev. 155, 122 (1967)

[10] J K Percus, Phys. Rev. A 9, 557 (1974)

[11] F Marchesoni and A Taloni, Phys. Rev. Lett. 97, 106101 (2006)

[12] A Taloni and M A Lomholt, Phys. Rev. E 78, 051116 (2008)

[13] M O Magnasco, Phys. Rev. Lett. 71 (1993) 1477

[14] T C Elston and C R Doering, J. Stat. Phys. 17, 359 (1996)

[15] R D Astumian and M Bier, Phys. Rev. Lett. 72, 1766 (1994)

[16] T Harms and R Lipowsky, Phys. Rev. Lett. 79, 2895 (1997)

[17] L Gao, X Luo, S Zhu and B Hu, Phys. Rev. E 67, 062104 (2003)

[18] D G Zarlenga, H A Larrondo, C M Arizmendi and F Family, Phys. Rev. E 65, 051101 (2007)

[19] Y Aghababaie, G I Menon and M Plischke, Phys. Rev. E 59 (1999) 2578

[20] I Goychuk and V Kharchenko, Phys. Rev. E 85 (2012) 051131

[21] V Kharchenko and I Goychuk, New J. Phys. 14 (2012) 043042

[22] C M Arizmendi and F Family, Physica A 232 (1996) 119

[23] C M Arizmendi and F Family, Physica A 251 (1998) 368

[24] J R Sanchez, F Family and C M Arizmendi, Phys. Lett. A 249, 281 (1998)

[25] M N Popescu, C M Arizmendi, A L Salas-Brito and F Family, Phys. Rev. Lett. 85, 3321 (2000)

[26] P Jung, J G Kissner and P Hänggi, Phys. Rev. Lett. 76, 3436 (1996) 